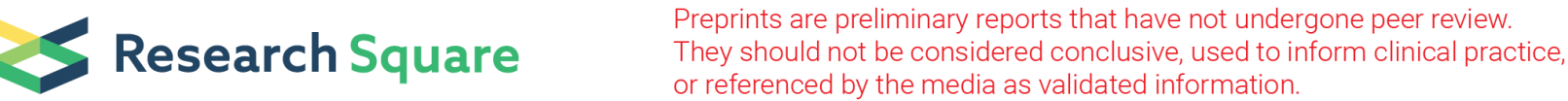

\section{A randomized study on the prophylactic use of acetaminophen to prevent fever after the removal of drainage tubes in lumbar surgery}

\section{Kaifeng Ye}

Peking University Third Hospital

\section{Yan Li}

Peking University Third Hospital

\section{Yong Xing}

Peking University Third Hospital

Kaixi Liu

Peking University Third Hospital

\section{Tengjiao Zhu}

Peking University Third Hospital

\section{Baichuan He}

Peking University Third Hospital

\section{Fang Zhou}

Peking University Third Hospital

Yun Tian ( $\nabla$ tiany@bjmu.edu.cn )

Peking University Third Hospital https://orcid.org/0000-0003-2310-6054

\section{Yanping Zhang}

Peking University Third Hospital

\section{Research article}

Keywords: lumbar drainage, fever, inflammation respond, prostaglandin, Acetaminophen

Posted Date: February 1st, 2021

DOI: https://doi.org/10.21203/rs.3.rs-141484/v2

License: (c) (1) This work is licensed under a Creative Commons Attribution 4.0 International License. Read Full License 


\section{Abstract}

Study design: a prospectively random clinical trial

Objective: To explore the efficacy of prophylactic use of Acetaminophen in preventing fever after the removal of tube drainage in lumbar surgery.

Summary and background data: Many inpatients encountered a fever in the first 24 hours when the drainage tube was removed. When fevers appeared, it was costly to exclude the possibility of deep infection and always failed to identify the etiology. Just like the postoperative fever, we suggested that the fever was caused by the normal inflammatory response, and tried to figure out whether the prophylactic use of Acetaminophen could lower the possibility of fever or not.

Methods: 183 consecutive patients undergoing lumbar spine surgery were prospectively randomized into 2 groups. 91 patients were allocated into the study group, they would receive a pill of Acetaminophen (Tylenol, 650mg) before the removal of lumbar tube drainage, and another one at $8 \mathrm{p} . \mathrm{m}$. The rest 92 patients were divided into the control group, they were treated in routine treatment without Acetaminophen. In the first 24 hours, a temperature higher than $37.7^{\circ} \mathrm{C}$ was defined as fever. The 2 groups were then compared for differences in age, gender, height, weight, body mass index (BMI), surgical segments, surgical time, blood loss, blood transfusion, American Society of Anesthesiologists (ASA) score, duration of the tube drainage, total volume of the drainage collection, variation of the levels of WBC, CRP, hospital stay since the removal of drainage to discharge and the rate of fever.

Results: There were no differences in all the basic information of 2 groups, but the fever rate of the 2 groups had significant differences $(P=0.006)$, the fever rate of the study group $(14 / 91,15.38 \%)$ was significant lower than the control group (30/92, 32.61\%).

Conclusion: Fever after the removal of tube drainage was caused by normal inflammation response, and a small dose of acetaminophen could significantly lower the possibility fever.

Trail registration: This trail was registered in the ClinicalTrial.gov PRS on July 13,2019 , and the ID was NCT04042948.

\section{Introduction}

Degenerative lumbar diseases are becoming increasingly common in the aging population, and the number of patients requiring surgery continues to increase[1]. Drainage tubes are widely used at the conclusion of spine surgery to prevent the hematoma formation, compression of the cauda equina nerves, severe neurological dysfunction and fever[2-4]. However, in our clinical work, many inpatients encountered a fever in the first 24 hours after the drainage tube was removed, and the percentage was approximately $40 \%$. When fevers appeared, we performed many laboratory tests to exclude the possibility of deep infection but failed to confirm the etiology of fever in most cases. The rate of surgical site 
infection has been reported to range from $0.7 \%$ to $12 \%[5-7]$, and the rate in our hospital was approximately $0.1 \%$. If an infection occurred, it would be difficult for a patient to recover. Fortunately, almost all the patients' temperatures gradually became normal after observation or antipyretic treatments before discharge. Similar to the reports of many previous studies, the evaluation of fever was costly, time consuming, and painful for patients and could add medical expenditure, prolong the hospital stay and waste health care resources[3, 4, 8-10].

To address this challenging issue, we carried out a thorough literature study. To the best of our knowledge, no previous research has focused on fever after the removal of drainage tubes. However, we found that many studies paid attention to early postoperative fever and suggested that the main reason for postoperative fever was the inflammatory response due to tissue trauma during surgery $[4,8,9,11]$. Inflammation is a normal physiological reaction during the process of healing, and the extent of tissue trauma correlates with the possibility of fever[8]. Many previous studies have further proven that the main inflammatory responses are due to pyrogenic cytokines, such as interleukin-I (IL-1), interleukin-6 (IL-6), tumor necrosis factor- $\alpha$ (TNF- $\alpha$ ), and interferon-gamma. These mediators act directly on the preoptic area of the hypothalamus and cause a release of prostaglandins, which could raise the thermoregulatory "set point" for body temperature and cause fever $[4,9,12,13]$. Both animal and human experiments have suggested that pretreatment with a cytokine antagonist could lower the concentration of pyrogenic cytokines and the possibility of fever $[12,14]$.

Furthermore, a previous investigation indicated that the presence of hematoma appeared to be a relatively common cause of fever[15]. In addition, many studies have discovered a high concentration of IL-6 in the drained blood after surgery $[12,16,17]$. Therefore, we infer that once the drainage tube is removed from lumbar patients after surgery, pyrogenic cytokines cannot flow outside of the surgical area and then accumulate gradually. A small hematoma forms, and cytokines can ooze into the circulation system to cause a fever. If we use acetaminophen to counteract the effects of prostaglandins in advance and then block the production of prostaglandins, perhaps we can lower the possibility of fever when the drainage tube is removed.

This prospective, randomized study was designed to determine the efficacy of the prophylactic use of acetaminophen before lumbar drainage tube removal. We chose Tylenol as the pretreatment medicine. We hypothesized that the preventive use of acetaminophen could lower the possibility of fever after the removal of drainage tubes in lumbar disease patients and shorten their stay in the hospital.

\section{Patients And Methods}

\section{Study design and patients}

We performed a single-blinded randomized controlled trial (NCT04042948) in the Department of Orthopaedics in our hospital from July 2019 to January 2020. Approvals were attained from the Ethics Committee of our hospital. 
Samples were collected from patients who underwent lumbar operations in our hospital. We calculated the sample size with a $5 \%$ significance level and $80 \%$ power, and 78 patients per group were necessary. We finally decided to recruit 95 patients in each group to allow for dropouts. The patients were randomly divided into 2 groups using a computer-generated random number list: the study group and the control group. The patients in the study group received an acetaminophen pill (Tylenol, $650 \mathrm{mg}$ ) before the removal of the lumbar drainage tube, and another pill was administered at 8 p.m., while the patients in the control group did not receive acetaminophen. In this study, we controlled the time of drainage tube removal to be from 8 a.m. to 10 a.m. in the morning to ensure that the first pill of acetaminophen was taken in the morning.

The inclusion criteria were as follows: 1 . adult patients conscious enough to provide informed consent; 2. clear diagnosis of degenerative lumbar spinal disease; 3. 1-2 surgical segments; 4 . indwelling drainage tube after the operation; and 5. normal hepatic function. The exclusion criteria were as follows: 1 . age < 18 years; 2 . refusal to participate; 3 . no indwelling drainage tube; 4 . more than 2 surgical segments; and 5. abnormal hepatic function.

\section{Protocol}

Our team was divided into four groups. The first group's responsibility was to screen and divide the patients into 2 groups, explain all the details to the patients and have them sign the conformed consent form if they agreed to participate. The second group comprised the attending physicians treating the patients. The third group collected clinical data, and the fourth group performed statistical analysis and wrote the manuscript.

All the included patients signed the conformed consent form on the day before the surgery, and the first group checked the computer-generated random number list, divided the patients into their corresponding groups and informed the second and third groups. The second and third groups were blinded to the patients' allocation before receiving information from the first group. The first group was blinded to all the clinical messages from the patients postoperatively.

All operations were performed by skilled surgeons under general anesthesia and fluoroscopy control on a radiolucent operation table. The extent of the surgery depended on the severity of the disease, and all the patients had a closed wound drain placed below the deep fascia over the exposed dura before wound closure. No complications were discovered during the operations.

All patients received traditional treatment and care before the drainage tubes were removed. Our nurses recorded the patient's daily temperature four times a day and the volume of the drainage collections once a day. All the data were uploaded to the medical system. When the recorded drainage volume of the in the last $24 \mathrm{~h}$ was below $50 \mathrm{ml}$ [18], the attending physician decided to remove the drainage tube. All patients underwent laboratory tests for the concentrations of white blood cells (WBCs) and C-reactive protein (CRP) the day after drainage tube removal. For the study group, the extra intervention was a pill of acetaminophen before the removal of the drainage tube and another pill taken orally at 8 p.m. Our nurse 
team kept recording the patients' temperatures four times a day and uploading the data to the medical system. The upper limit of a normal temperature was $37.7^{\circ} \mathrm{C}[11]$, so a temperature $>37.8^{\circ} \mathrm{C}$ at any time within the first $24 \mathrm{~h}$ after the removal of the drainage tube indicated that a fever developed.

\section{Data and observation}

All the patients' basic clinical information was collected, including age, sex, height, weight, body mass index (BMI), surgical segments, surgical time, blood loss, blood transfusion, American Society of Anesthesiologists (ASA) score, duration of drainage tube use, and total drainage volume.

The main observation index was temperature within the first $24 \mathrm{~h}$ after the drainage tube was removed. Once a fever developed, the data collection of that patient ended. The attending physician would start the diagnostic workups depending on the medical history and physical examination to identify the etiology of fever. The possible workups included chest X-ray, urinalysis, urine culture, and blood culture. The other observation indexes were the variations in WBC and CRP levels, the length of hospital stay from the removal of the drainage tube to discharge and the whole hospital stay.

\section{Statistical analysis}

A descriptive analysis was performed of the main study variables. All continuous data were tested for normality with the Shapiro-Wilk test. If the data were normally distributed, Student's $t$ test was used for analysis. The Mann-Whitney U test was performed for non-normally distributed data. For ranked data, the chi-square test was used for analysis. $P<0.05$ was considered statistically significant. All analyses were performed with SPSS Statistics (version 24.0; IBM Corp., Armonk, NY, USA).

\section{Results}

The study process is shown in Figure 1. In total, 190 patients participated in this research, and 7 patients were excluded because they refused the operation (6 patients) or underwent reoperation for postoperative hematoma (1 patient). Finally, the study comprised 183 patients; 91 patients were in the study group, and the rest were in the control group.

There were 80 males and 103 female patients, and the average age was 51.34 years. The average weight was $69.80 \mathrm{~kg}$, and the average height was $165.53 \mathrm{~cm}$, equivalent to an average BMI of $25.46 \mathrm{~kg} / \mathrm{m}^{2}$. The characteristics of the basic medical information are listed in Table 1.

Table 1 The characteristics of the 2 groups considered continuous data. 


\begin{tabular}{|c|c|c|c|c|}
\hline Characteristic & $\begin{array}{c}\text { Study } \\
\text { group } \\
\text { Mean (SD) }\end{array}$ & Control group & Total & $\bar{P}$ \\
\hline Age (y) & $\begin{array}{c}50.76 \\
(11.70)\end{array}$ & $51.791(12.12)$ & $\begin{array}{c}51.34 \\
(11.89)\end{array}$ & 0.419 \\
\hline Height (cm) & $\begin{array}{c}164.79 \\
(8.39)\end{array}$ & $166.26(8.27)$ & $\begin{array}{c}165.53 \\
(8.34)\end{array}$ & 0.230 \\
\hline Weight (kg) & $\begin{array}{c}69.34 \\
(11.34)\end{array}$ & $70.25(10.31)$ & $\begin{array}{c}69.80 \\
(10.81)\end{array}$ & 0.571 \\
\hline BMI $(\mathrm{kg} / \mathrm{m} 2)$ & $\begin{array}{l}25.52 \\
(3.67)\end{array}$ & $25.39(3.01)$ & $\begin{array}{l}25.46 \\
(3.35)\end{array}$ & 0.957 \\
\hline Surgical segments & $1.29(0.48)$ & $1.35(0.55)$ & $\begin{array}{l}1.32 \\
(0.51)\end{array}$ & 0.468 \\
\hline Surgical time (min) & $\begin{array}{l}124.44 \\
(37.17)\end{array}$ & $\begin{array}{l}121.81 \\
(39.24)\end{array}$ & $\begin{array}{l}123.19 \\
(38.07)\end{array}$ & 0.649 \\
\hline Blood loss (ml) & $\begin{array}{c}246.39 \\
(143.23)\end{array}$ & $\begin{array}{c}266.00 \\
(145.62)\end{array}$ & $\begin{array}{c}256.19 \\
(144.36)\end{array}$ & 0.307 \\
\hline lood transfusion (ml) & 90.83 & 109.24 & 99.46 & 0.071 \\
\hline ll drainage volume (ml) & $\begin{array}{c}481.43 \\
(302.60)\end{array}$ & $\begin{array}{c}454.99 \\
(324.47)\end{array}$ & $\begin{array}{c}468.21 \\
(313.13)\end{array}$ & 0.402 \\
\hline Iration of drainage (d) & $3.36(1.01)$ & $3.30(0.91)$ & $\begin{array}{l}3.33 \\
(0.96)\end{array}$ & 0.867 \\
\hline Hospital stay (d) & $7.68(1.93)$ & $8.12(2.71)$ & $\begin{array}{l}7.91 \\
(2.36)\end{array}$ & 0.517 \\
\hline WBC $\left(* 10^{\wedge} 9 / \mathrm{L}\right)$ & $8.25(2.68)$ & $8.69(2.78)$ & $\begin{array}{l}8.47 \\
(2.73)\end{array}$ & 0.244 \\
\hline CRP (mg/dL) & $3.77(3.61)$ & $4.09(3.58)$ & $\begin{array}{l}3.93 \\
(3.58)\end{array}$ & 0.402 \\
\hline $\begin{array}{l}\text { E hospital stay after removal of } \\
\text { he drainage tube (d) }\end{array}$ & $2.23(1.42)$ & $2.82(2.61)$ & $\begin{array}{r}2.52 \\
(2.12) \\
\end{array}$ & 0.151 \\
\hline
\end{tabular}

The Shapiro-Wilk (Table 2) test showed that other than weight, the remaining continuous data were nonnormally distributed $(\mathrm{P}<0.05)$, so we analyzed them with the Mann-Whitney $\mathrm{U}$ test, while the weight data were tested by Student's $t$ test. The comparison of the basic data is displayed in Tables 1 and 3 . We determined that there were no differences in age, sex, height, weight, BMI, surgical segments, surgical time, blood loss, transfusion, ASA score, duration of drainage tube use, or total drainage volume between the two groups $(P>0.05)$. Although the levels of WBC and CRP were lower, length of hospital stay and the hospital after the removal of drainage tube was shorter in the study group than in the control group, the differences were not significant $(P>0.05)$. However, the rate of fever was significantly different $(P=0.006)$, and the study group $(14 / 91,15.38 \%)$ had a lower percentage of fever than the control group (30/92, $32.61 \%)$.

Table 2 The Shapiro-Wilk test for normality of the continuous data $(\mathrm{P}>0.05$ suggests that the data were normally distributed) 


\begin{tabular}{ccc}
\hline Characteristic & \multicolumn{2}{c}{ P } \\
\cline { 2 - 3 } & Study group & Control group \\
\hline Age & 0.001 & 0.004 \\
Height & 0.020 & 0.206 \\
Weight & 0.620 & 0.213 \\
BMI & 0.039 & 0.118 \\
\hline Surgical segments & 0.000 & 0.000 \\
\hline Surgical time & 0.006 & 0.000 \\
Blood loss & 0.000 & 0.000 \\
Blood transfusion & 0.000 & 0.000 \\
Total drainage volume & 0.006 & 0.000 \\
Duration of drainage & 0.000 & 0.000 \\
\hline Duration of hospital stay & 0.000 & 0.000 \\
WBC & 0.000 & 0.000 \\
\hline CRP & 0.000 & 0.000 \\
\hline n of hospital stay after removal of the drainage tube & 0.000 & 0.000 \\
\hline
\end{tabular}

Table 3 The comparison of ranked data

\begin{tabular}{ccccc}
\hline Characteristic & Study group & Control group & Total & P \\
\hline Sex (Male/Female) & $39 / 52$ & $41 / 51$ & $80 / 103$ & 0.816 \\
\hline ASA score (I/II) & $48 / 43$ & $49 / 43$ & $97 / 86$ & 0.945 \\
\hline Segments (One/Two) & $66 / 25$ & $63 / 29$ & $129 / 64$ & 0.548 \\
ever rate (Fever/Normal) & $14 / 77$ & $30 / 62$ & $44 / 139$ & 0.006 \\
\hline
\end{tabular}

Among the cases of fever, there were 3 positive urinary cultures, 1 in the study group (Escherichia coli) and 2 in the control group (Acinetobacter junii and coagulase negative staphylococcus), which were treated by antibiotics depending on the drug sensitivity test. Additionally, 2 patients with fever encountered cerebrospinal fluid leakage (discovered after the postoperative observation). During followup in the outpatient department, no infections were discovered after discharge.

\section{Discussion}

Similar to postoperative fever, fever after the removal of a drainage tube is always a challenge for many doctors. The diagnostic workups for fever could be costly, waste time, and prolong the hospital stay of patients. Moreover, most of the evaluations failed to identify the cause of fever. In this study, we used acetaminophen (Tylenol) as a prophylactic treatment for fever after the removal of drainage tubes in lumbar surgery, and the results showed that this approach could significantly lower the possibility of fever.

To the best of our knowledge, our research was the first to focus on the problem of fever after the removal of a drainage tube placed during lumbar surgery. Many previous studies have focused on postoperative fever. The most acceptable conclusion was that fever was the physiological response due to the damage 
caused by surgical trauma, and the degree of fever was correlated with the extent of tissue trauma during surgery $[4,8,11,13]$. The inflammation reaction stimulated by tissue trauma could trigger the release of cytokines such as IL1, IL6 and TNF-a in the local surgical area, and the released cytokines acted as endogenous pyrogens to stimulate the preoptic area of the hypothalamus to increase prostaglandin production, raising the thermoregulatory set point for body temperature $[9,13,14,19,20]$. Based on these theories, we proposed that when the drainage tube was removed, the local inflammatory cytokines could not flow out, and then a small hematoma formed. With the increase in pressure caused by the hematoma and the concentration of cytokines, the inflammatory cytokines could penetrate the vessel circulation and then raise the body temperature. Therefore, if we use acetaminophen in advance to depress the function of prostaglandin (Figure 4), we could lower the possibility of fever when the drainage tube is removed. The results of the study proved our hypothesis.

Many previous studies could directly or indirectly support our proposal. The reports of Chmel et al [21] and Gemer et al [22] both suggested that hematoma of the wound site was the source of fever, which was in line with our thoughts. Many previous studies have stated that the postoperative drainage fluid is rich in inflammatory cytokines such as IL1, IL6 and TNF-a, and the levels of these cytokines are higher in fever patients $[12,16,17]$. Mark et al [23] and Gideon et al [8] both compared the fever rate of patients with or without drainage tubes postoperatively and declared that patients without postoperative drainage were more vulnerable to fever. More interesting, learning from Hao's [18] research, we found that if the drainage tube is intermittently clamped after lumbar surgery, the patient was more likely to have fever. All the above conclusions could support our original idea that the removal of the drainage tube could stop the outflow of inflammatory cytokines and then cause fever.

Tylenol, a brand of acetaminophen, is a common analgesic-antipyretic medicine in our clinical treatment. This drug could inhibit prostaglandin synthase in the hypothalamic thermoregulation center and then reduce the production of prostaglandin. Based on what we discussed above, when the drainage tube was removed, the elevated cytokines could act on the hypothalamic thermoregulation center and cause a fever. The prophylactic medicine acetaminophen could depress the activity of prostaglandin synthase to fight against stimulation from the cytokines to synthase, and then lower the possibility of fever. The halflife of Tylenol is approximately 2 hours, so the serum concentration of acetaminophen would be too low to have an antipyretic effect the next day. It was not necessary to worry if the use of acetaminophen would mask signs of infection, and the number of infectious cases in the study group could prove this.

The concentrations of WBCs and CRP were not significantly different between groups in this study. As we observed, acetaminophen could only depress the activity of prostaglandin synthase but not suppress the inflammatory reaction caused by surgical trauma, so it was reasonable to accept this result. The length of hospital stay after drainage removal of the study group was shorter than that of the control group ( 2.23 days vs 2.82 days), but the difference was not significant because some patients asked to delay their discharge due to weekends or their personal condition. 
Our study had some limitations. First, this was not a triple-blinded study. Second, due to limited funding, we did not directly test the changes in the concentrations of cytokines before and after the removal of the drainage tube. However, we were the first to focus on fever after the removal of drainage tubes for lumbar surgery, and our research proved that prophylactic treatment with acetaminophen could significantly lower the potential of developing fever.

\section{Conclusion}

Fever after the removal of drainage tubes was caused by a normal inflammatory response. After the removal of the drainage tube from lumbar patients, a small dose of acetaminophen could significantly lower the possibility of fever. This approach could improve patient comfort, reduce the wasting of medical resources and speed up the discharge of patients. In our further research, we will try to determine the risk factors for fever after drainage tube removal and narrow the scope of patients who should receive prophylactic medicine.

\section{Abbreviations}

IL-1: interleukin-l; IL-6: interleukin-6; TNF- $\alpha$ : tumor necrosis factor- $\alpha$; WBCs: white blood cells; CRP: Creactive protein; BMI: body mass index; ASA: American Society of Anesthesiologists;

\section{Declarations}

\section{Acknowledgements}

We thank Rui Wang and other nurses for the help to this study.

\section{Authors' contributions}

Kaifeng Ye and Yan Li collected data and write the article; Yong Xing, Tengjiao Zhu and Baichuan He were responsible for literature review; Kaixi Liu drew the illustrations; Fang Zhou, Yanping Zhang and Yun Tian designed the subject. All authors read and approved the final manuscript.

\section{Funding}

This work was supported by the National Key R\&D Program of China (2018YFE0104200).

\section{Availability of data and materials}

All data and materials used to support the findings of this study are included within the article.

\section{Ethics approval and consent to participate}

All procedures performed in this study were in accordance with the ethical standards of World Medical Association Declaration of Helsinki Ethical Principles for Medical Research Involving Human Subjects. 
The Ethics Committee of Peking University Third Hospital approved this subject.

\section{Consent for publication}

All authors agree to publish "A randomized study on the prophylactic use of acetaminophen to prevent fever after the removal of drainage tubes in lumbar surgery" in Journal of Orthopaedic Surgery and Research.

\section{Competing interests}

We declare that we do not have any commercial or associative interest that represents a conflict of interest in connection with the work submitted.

\section{References}

1. Vos T, Flaxman AD, Naghavi M, et al. (2012) Years lived with disability (YLDs) for 1160 sequelae of 289 diseases and injuries 1990-2010: a systematic analysis for the Global Burden of Disease Study 2010. Lancet 380:2163-2196. doi: 10.1016/s0140-6736(12)61729-2

2. Liu JM, Chen WZ, Fu BQ, et al. (2016) The Use of Closed Suction Drainage in Lumbar Spinal Surgery: Is It Really Necessary? World Neurosurg 90:109-115. doi: 10.1016/j.wneu.2016.02.091

3. Yoo JH, Kim KT, Kim TY, et al. (2017) Postoperative fever after hemiarthroplasty in elderly patients over 70 years of age with displaced femoral neck fracture: Necessity of routine workup? Injury 48:441-446. doi: 10.1016/j.injury.2016.12.013

4. Mayo BC, Haws BE, BohI DD, et al. (2018) Postoperative Fever Evaluation Following Lumbar Fusion Procedures. Neurospine 15:154-162. doi: 10.14245/ns.1836026.013

5. Pull ter Gunne AF, van Laarhoven CJ, Cohen DB (2010) Incidence of surgical site infection following adult spinal deformity surgery: an analysis of patient risk. Eur Spine J 19:982-988. doi:

10.1007/s00586-009-1269-1

6. Tamai K, Wang C, Heindel P, et al. (2019) Perioperative Catheter Use as a Risk Factor for Surgical Site Infection After Cervical Surgery: An Analysis of 39,893 Patients. Spine (Phila Pa 1976) 44:E157-e161. doi: $10.1097 /$ brs. 0000000000002790

7. Gu W, Tu L, Liang Z, et al. (2018) Incidence and risk factors for infection in spine surgery: A prospective multicenter study of 1764 instrumented spinal procedures. Am J Infect Control 46:8-13. doi: 10.1016/j.ajic.2017.09.025

8. Blumstein GW, Andras LM, Seehausen DA, et al. (2015) Fever is common postoperatively following posterior spinal fusion: infection is an uncommon cause. J Pediatr 166:751-755. doi:

10.1016/j.jpeds.2014.11.033

9. Maday KR, Hurt JB, Harrelson P, et al. (2016) Evaluating postoperative fever. Jaapa 29:23-28. doi: 10.1097/01.JAA.0000496951.72463.de 
10. Ashley B, Spiegel DA, Cahill P, et al. (2017) Post-operative fever in orthopaedic surgery: How effective is the 'fever workup?'. J Orthop Surg (Hong Kong) 25:2309499017727953. doi: $10.1177 / 2309499017727953$

11. Seo J, Park JH, Song EH, et al. (2017) Postoperative Nonpathologic Fever After Spinal Surgery: Incidence and Risk Factor Analysis. World Neurosurg 103:78-83. doi: 10.1016/j.wneu.2017.03.119

12. Andres BM, Taub DD, Gurkan I, et al. (2003) Postoperative fever after total knee arthroplasty: the role of cytokines. Clin Orthop Relat Res:221-231. doi: 10.1097/01.blo.0000093914.26658.55

13. Narayan M, Medinilla SP (2013) Fever in the postoperative patient. Emerg Med Clin North Am 31:1045-1058. doi: 10.1016/j.emc.2013.07.011

14. Kahokehr A, Hill AG (2011) Early post-operative fever. ANZ J Surg 81:5-6. doi: 10.1111/j.14452197.2010.05611.x

15. Hausmann MJ, Kachko L, Basok A, et al. (2009) Prolonged fever following kidney biopsy: a case report. Int Urol Nephrol 41:423-425. doi: 10.1007/s11255-008-9486-9

16. Handel M, Winkler J, Hornlein RF, et al. (2001) Increased interleukin-6 in collected drainage blood after total knee arthroplasty: an association with febrile reactions during retransfusion. Acta Orthop Scand 72:270-272. doi: 10.1080/00016470152846600

17. Athanassious C, Samad A, Avery A, et al. (2011) Evaluation of fever in the immediate postoperative period in patients who underwent total joint arthroplasty. J Arthroplasty 26:1404-1408. doi: 10.1016/j.arth.2011.02.019

18. Hao QY, Liu CY, Fu CJ, et al. (2016) Improved Intermittent-clamped Drainage in Lower Lumbar Internal Fixation: A Randomized Prospective Study. Chin Med J (Engl) 129:2804-2809. doi: 10.4103/0366-6999.194639

19. Dionigi R, Dionigi G, Rovera F, et al. (2006) Postoperative fever. Surg Infect (Larchmt) 7 Suppl 2:S1720. doi: $10.1089 /$ sur.2006.7.s2-17

20. Burke L (2010) Postoperative fever: a normal inflammatory response or cause for concern. J Am Acad Nurse Pract 22:192-197. doi: 10.1111/j.1745-7599.2010.00492.x

21. Chmel H, Palmer JA, Eikman EA (1988) Soft tissue hematoma as a cause of fever in the adult. Diagn Microbiol Infect Dis 11:215-219. doi: 10.1016/0732-8893(88)90007-7

22. Gemer O, Shenhav S, Segal S, et al. (1999) Sonographically diagnosed pelvic hematomas and postcesarean febrile morbidity. Int J Gynaecol Obstet 65:7-9. doi: 10.1016/s0020-7292(99)00003-x

23. Brown MD, Brookfield KF (2004) A randomized study of closed wound suction drainage for extensive lumbar spine surgery. Spine (Phila Pa 1976) 29:1066-1068. doi: 10.1097/00007632-20040515000003

\section{Figures}




\section{Calculated sample size: 156 patients}

Allow for dropouts

\section{Actual recruitment:}

190 patients

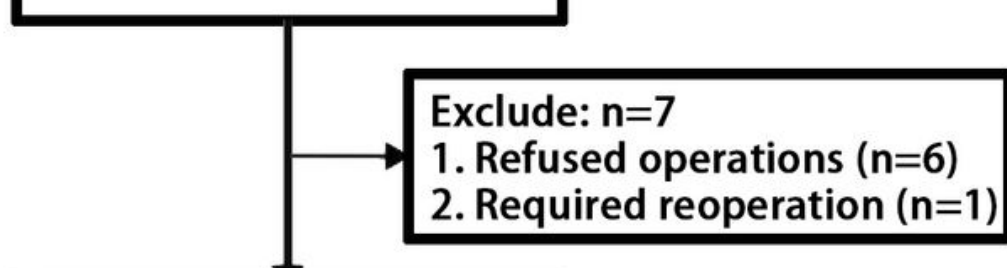

\section{Randomized distribution $^{(n=183)}$}

Assign to the study group: $\mathrm{n}=91$

1. Take a pill of Tylenol before the removl of drainage

2. Take another pil at 8 p.m on the same day
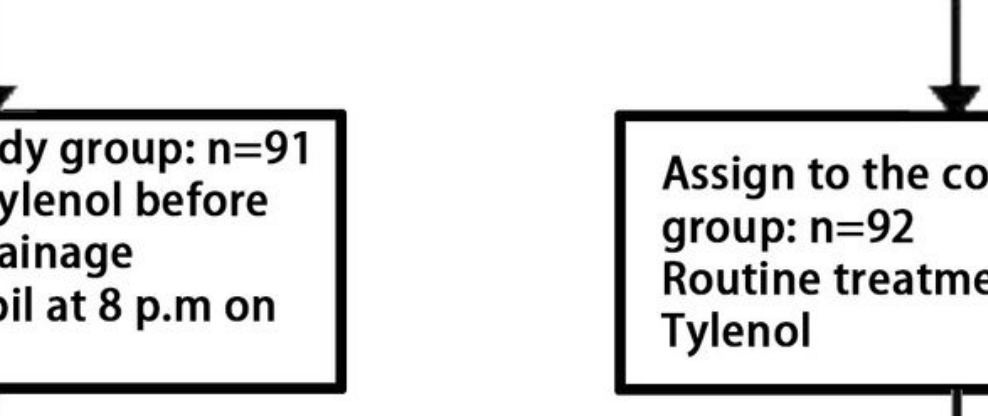

Assign to the control

group: $\mathrm{n}=92$

Routine treatment without

Tylenol

\section{Figure 1}

The process of the study 


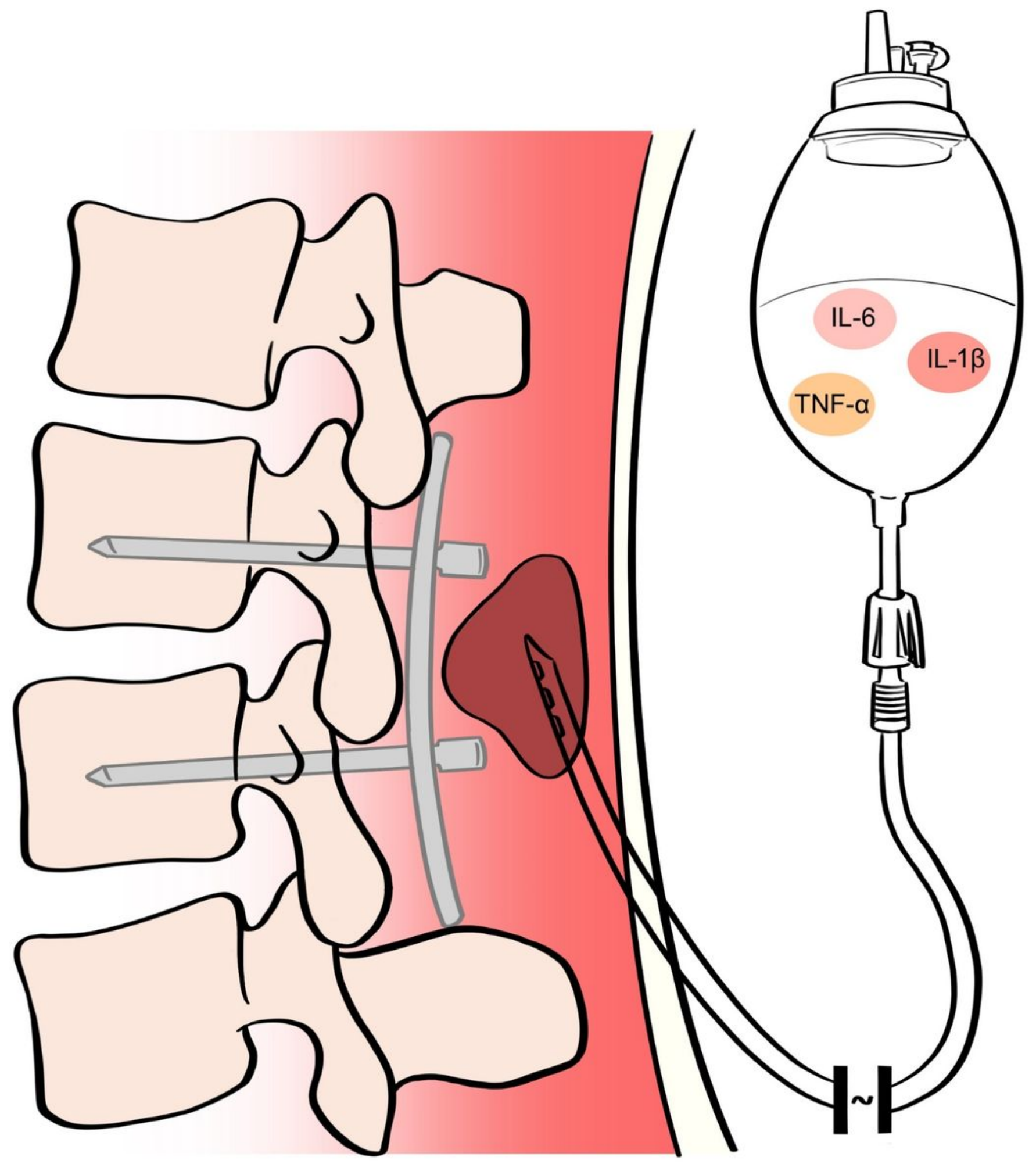

Figure 2

The role of drainage tubes in preventing fever after lumbar surgery. 


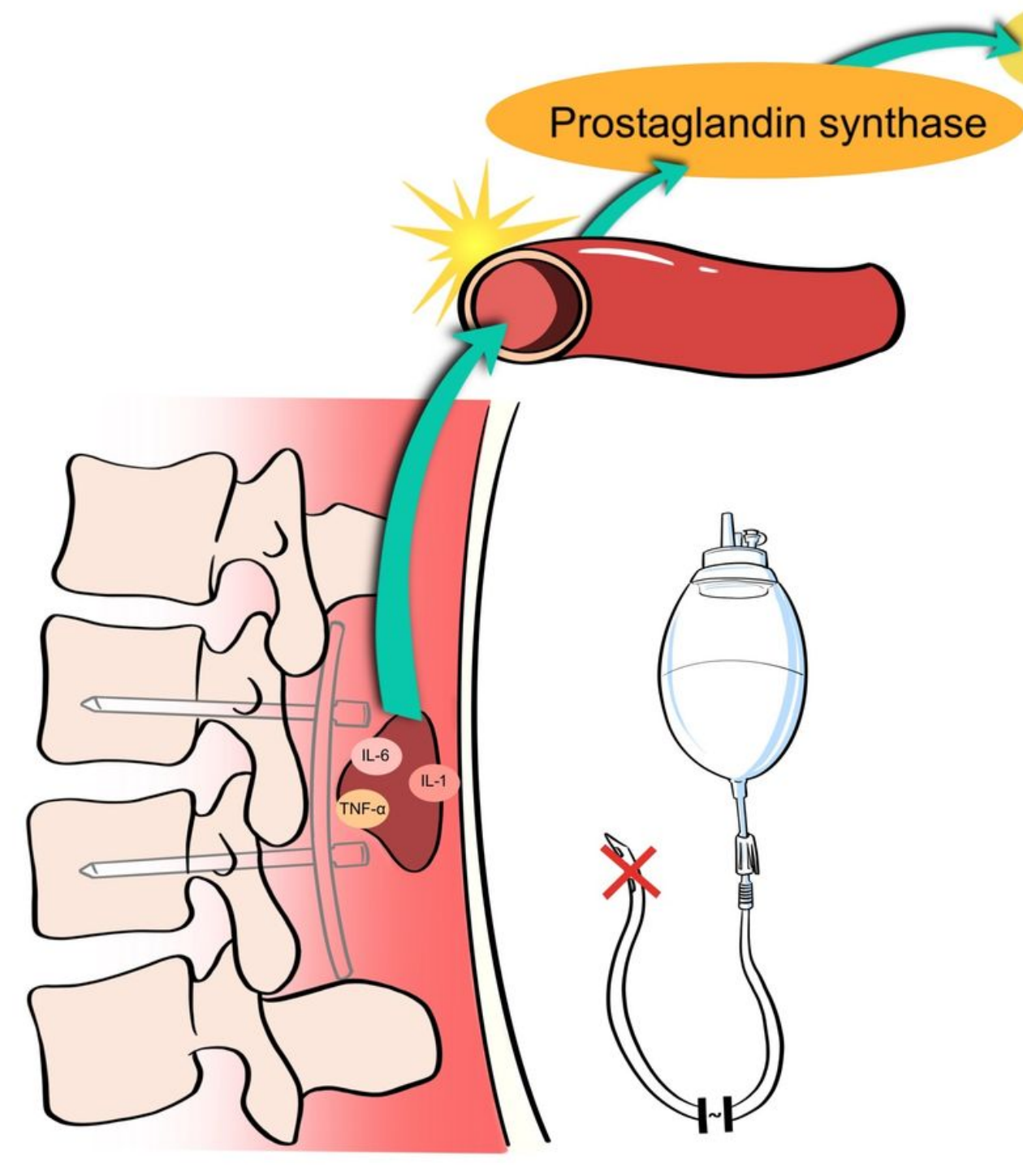

$P G$

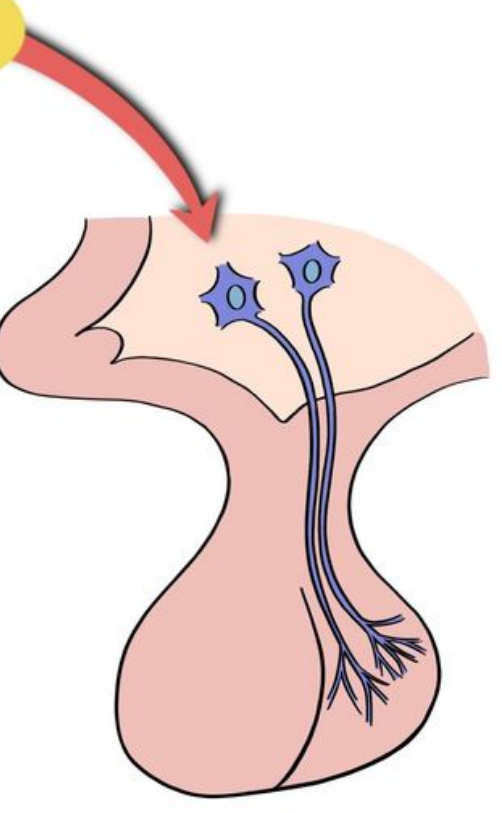

Figure 3

The mechanism of fever after the removal of drainage tubes in lumbar surgery. (PG: prostaglandin) 


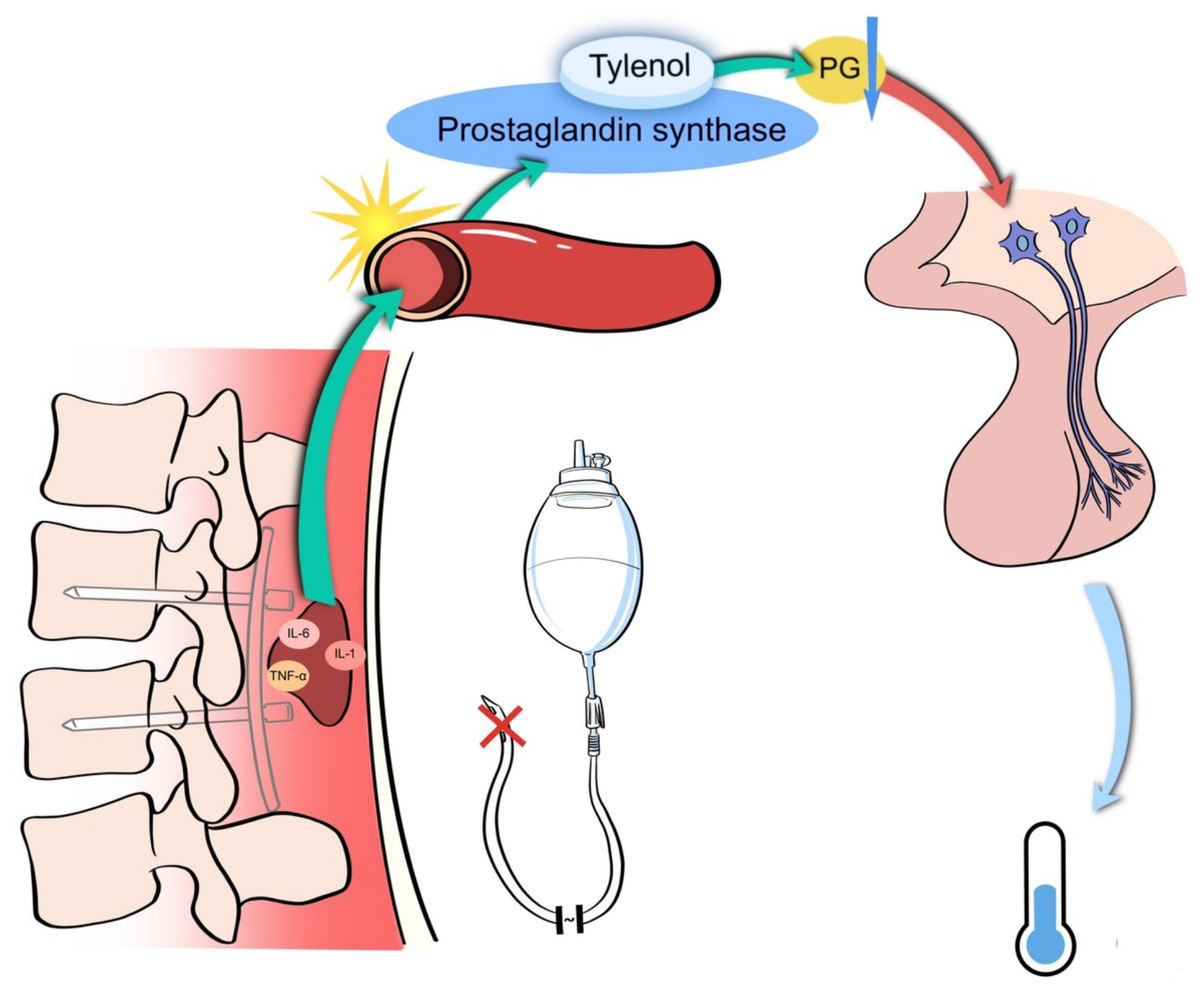

Figure 4

The mechanism of acetaminophen in preventing fever after the removal of the drainage tube. (PG: prostaglandin) 